Research Paper Number 921

\title{
Transactions Costs and Portfolio Choice in a Discrete-Continuous Time Setting
}

\author{
Darrell Duffie and Tong-sheng Sun \\ Forthcoming: Journal of Economic Dynamics and Control
}

November, 1986; Revised: July, 1989

\begin{abstract}
This paper makes the following observation concerning a new formulation of the consumption and portfolio choice model of Merton (1971), with transactions costs. Suppose an investor observes his or her current wealth only when making a transaction, that transactions are costly, and that decisions to transact can be made at any time based on all current information. If, at each transaction, the agent is charged a fixed fraction of current portfolio value, an optimal policy exists and the optimal interval of time between transactions is fixed, independent of time and current wealth.
\end{abstract}

We thank Monique Pontier, Monique Jeanblanc-Picqué, and a referee for many suggestions and comments. Correspondence should be directed to Darrell Duffie at the Graduate School of Business, Stanford University, Stanford CA 94305-5015. This paper was written when Tong-sheng Sun, currently with Shearson Lehman Hutton, was at Stanford University. The paper reflects the views of the authors and should not be interpreted as reflecting those of Shearson Lehman Hutton. This is in the Research Paper Series, Graduate School of Business, Stanford University. 


\section{INTRODUCTION}

This paper makes a modest observation concerning a new formulation of the consumption and portfolio choice model of Merton (1971), with transactions costs. Suppose the agent observes his or her current wealth only when making a transaction, that transactions are costly, and that the decision to transact can be made at any time based on all current information. If, at each transaction, the agent is charged a fixed fraction of current wealth, the optimal interval of time between transactions is fixed, independent of time and current wealth. We call this a discretecontinuous time formulation.

Since this paper was submitted for publication, Davis and Norman (1988) have presented an elegant and natural solution of Merton's problem with proportional transactions costs. As far as the volume and timing of trade, of course, the direct continuous-time models of Merton as well as Davis and Norman are not robust to lump sum transactions costs. They both call for an infinite number of transactions during an arbitrarily small interval of time. Our simple solution, on the other hand, is based on lump sum transactions costs proportional to portfolio value, a restrictive assumption. For further results on continous-time portfolio choice with transactions costs, see Leland (1985) and Constantinides (1986).

This paper is organized as follows. Section II describes the setting. In general, the optimal intervals of time between investment decisions are not independent of current wealth. Nevertheless, it is shown in Section III that the optimal intervals are equal constants when the investor is of constant relative risk aversion and there are "portfolio management fees" as well as proportional transaction costs. The discrete-time theory can therefore be thought of as a special case of the continuoustime theory with transactions costs. Section IV extends the model to a market with $m$ risky securities, and an analogous conclusion follows.

\section{MODEL}

Uncertainty. Let $B=\left\{B_{t}: t \geq 0\right\}$ be a Standard Brownian Motion on its standard filtered probability space $(\Omega, \mathcal{F}, \mathbf{F}, \mathcal{P})$. The filtration $\mathbf{F}=\left\{\mathcal{F}_{t}: t \geq 0\right\}$ of $\sigma$-algebras represents information revealed by the Brownian Motion. 
Security markets. There are two securities in the economy: one riskless, one risky. The risky security has a strictly positive price process $S^{(1)}$ and a cumulative dividend process $D$ that satisfy

$$
S_{t}^{(1)}+D_{t}=1+\int_{0}^{t} S_{s}^{(1)} \mu d s+\int_{0}^{t} S_{s}^{(1)} \sigma d B_{s}, \quad t \geq 0,
$$

for given positive scalars $\mu$ and $\sigma$. The price process of the riskless security is

$$
S_{t}^{(0)}=1+\int_{0}^{t} r S_{s}^{(0)} d s, \quad t \geq 0
$$

for some interest rate $r>0$.

There is a medium of exchange and numeraire in the economy, say money. Only money is exchangeable for consumption; this is the so-called Clower constraint. Money cannot be borrowed, it can only be acquired by selling the securities. Clearly, there is no investment demand for money because there exists a riskless security with a positive interest rate. Money holdings at time $t$ are denoted $M_{t}$. One unit of money can be exchanged at any time for one unit of consumption. For simplicity, the investor is assumed to receive no further income from non-capital sources, and starts with the initial stock of money $M_{0}=0$.

A (portfolio) transaction consists of withdrawing wealth in the form of money from the investment in securities and adjusting the portfolio of securities. Trading opportunities are available continuously in time, but not costlessly.

Transactions costs. Transactions costs are incurred when information is processed and a portfolio transaction is made. There are two forms of transactions costs: withdrawal costs and portfolio management fees. Withdrawal costs are assumed to be affine; that is, the investor pays fixed plus proportional costs of the amount of money withdrawn. In addition, the investor pays a fraction $\epsilon>0$ of the total wealth in securities at the beginning of each interval, a portfolio management fee. The portfolio management fee is meant to include the cost of adjusting the portfolio and the cost of processing information.

For example, if the total wealth in securities immediately before a transaction at time $t$ is $X_{t}$ and an amount of money $W_{t}$ is withdrawn, the total transactions cost is $a W_{t}+b+\left(X_{t}-W_{t}\right) \epsilon$, for non-negative constants $a, b$, and $\epsilon$. 
Information. We can interpret $\mathcal{F}_{t}$ as the information available at time $t$. Given the structure of transactions costs, consumption and investment decisions are, however, optimally made at intervals. During each interval there is no transaction. All interim dividends of the risky security are re-invested continually in the risky security, and all interim interest income is re-invested continually in the riskless security. Since there is a lump sum cost for processing each transaction, a continually varying trading strategy would involve infinite costs. The investor therefore chooses (possibly random) instants of time at which to process information and make consumption and investment decisions. In other words, even though information is available continuously through the filtration $\left\{\mathcal{F}_{t}: t \geq 0\right\}$, the investor, in the presence of decision costs, chooses to receive information via a controllable filtration $\mathbf{H}=\left\{\mathcal{H}_{t}: t \geq 0\right\}$, with $\mathcal{H}_{t}=\mathcal{F}_{\tau_{k}}, t \in\left[\tau_{k}, \tau_{k+1}\right)$, where $\tau_{k}$ is a $\mathcal{H}_{\tau_{k-1}}$-measurable stopping time at which the $k$-th transaction occurs. The filtration $\mathbf{H}$ is controllable in the sense that the investor is allowed to choose any sequence $\tau=\left\{\tau_{k}: k=1,2,3, \ldots\right\}$ of such transaction times with $\tau_{0} \equiv 0$. Let $T=\left\{T_{k} \equiv \tau_{k+1}-\tau_{k}: k=1,2,3, \ldots\right\}$ denote the corresponding sequence of transaction intervals . Finding an optimal stopping policy $\tau$ is clearly equivalent to finding an optimal interval policy $T$.

Preferences. The consumption space $\mathbf{C}$ for the investor consists of positive $\mathbf{H}-$ adapted consumption processes $C=\left\{C_{t}: t \geq 0\right\}$ satisfying $\int_{0}^{t} C_{s} d s<\infty$ almost surely for all $t \geq 0$ and

$$
U(C) \equiv E\left[\int_{0}^{\infty} e^{-\rho t} u\left(C_{t}\right) d t\right]<\infty
$$

where $\rho$ is a strictly positive scalar discount factor and $u(c)=c^{\alpha} / \alpha$ for some $\alpha \in(0,1)$. The investor has the utility function $U$ defined by $(3)$.

Feasible policies. Let $V_{\tau_{k}}$ be the market value of the investment in the risky security chosen at time $\tau_{k}$. Let $\mathbf{T}$ denote the space of sequences of strictly positive transaction intervals; $\mathbf{W}$, the space of positive $\mathbf{H}$-adapted money withdrawal processes; and $\mathbf{V}$, the space of $\mathbf{H}$-adapted investment processes for the risky security. A policy is a quadruplet $(T, V, W, C) \in \mathbf{T} \times \mathbf{V} \times \mathbf{W} \times \mathbf{C}$.

We need to characterize budget feasible policies. Given a policy $(T, V, W, C)$, 
the money holding at any time $t$ is

$$
M_{t}=\sum_{\left\{k: \tau_{k} \leq t\right\}}\left[(1-a) W_{\tau_{k}}-b\right]-\int_{0}^{t} C_{s} d s .
$$

Let $X\left(\tau_{k}\right)$ denote the total wealth invested in securities at time $\tau_{k}$, before the $k$-th transaction. Of this amount, $W\left(\tau_{k}\right)$ is withdrawn, and a fraction $\epsilon$ of the remainder, $X\left(\tau_{k}\right)-W\left(\tau_{k}\right)$, is paid in management fees, leaving $Z\left(\tau_{k}\right)=(1-$ $\epsilon)\left[X\left(\tau_{k}\right)-W\left(\tau_{k}\right)\right]$ for re-investment. Of this, $V\left(\tau_{k}\right)$ is invested in the risky asset, with a per-dollar payback of $\Gamma_{k+1}$ at the next transaction date, including continually reinvested dividends. Based on (1) and Ito's Lemma,

$$
\Gamma_{k+1}=\exp \left[\left(\mu-\frac{1}{2} \sigma^{2}\right) T_{k}+\sigma\left(B_{\tau_{k+1}}-B_{\tau_{k}}\right)\right] .
$$

The remainder, $Z\left(\tau_{k}\right)-V\left(\tau_{k}\right)$, is invested risklessly at the continuously compounding interest rate $r$. The total wealth invested at the time of the $(k+1)$-th transaction is therefore

$$
\begin{aligned}
X\left(\tau_{k+1}\right) & =\left[Z\left(\tau_{k}\right)-V\left(\tau_{k}\right)\right] e^{r T_{k}}+V\left(\tau_{k}\right) \Gamma_{k+1} \\
& =(1-\epsilon)\left[X\left(\tau_{k}\right)-W\left(\tau_{k}\right)\right] e^{r T_{k}}+V\left(\tau_{k}\right)\left(\Gamma_{k+1}-e^{r T_{k}}\right)
\end{aligned}
$$

Since $M_{0}=0, X_{0}$ is the initial wealth endowment for the investor. The policy $(T, W, V, C)$ is budget feasible if the associated invested wealth process $X$ of $(6)$ and money process $M$ of (4) are non-negative. If $b \neq 0$, the appropriate definition of the non-negative wealth constraint is a bit delicate, but all of our formal results apply to the case $b=0$.

The control problem for the investor is thus summarized by:

$$
\mathbf{U}\left(X_{0}\right) \equiv \sup _{(T, W, V, C) \in \Phi} E\left[\int_{0}^{\infty} e^{-\rho t} u\left(C_{t}\right) d t\right]
$$

where $\Phi$ denotes the space of budget feasible policies.

Technical conditions. In solving the control problem, we will restrict the parameters $(\mu, \sigma, \rho, \alpha, a, b, \epsilon)$ in order to guarantee the existence of a solution. A precise restriction will be stated when necessary. 


\section{MAIN RESULTS}

Because there is no investment demand for money, it is not optimal for the investor to withdraw more money than the amount needed for consumption before the next transaction. This is proved as Lemma 1 in Appendix 1. An optimal policy must therefore satisfy

$$
\int_{\tau_{k}}^{\tau_{k+1}} C_{t} d t=(1-a) W_{\tau_{k}}-b, \quad k=1,2,3, \ldots
$$

This implies that $M_{\tau_{k}}=(1-a) W_{\tau_{k}}-b$ for all $\tau_{k}$. The control problem (7) is therefore equivalent to

$$
\mathbf{U}\left(X_{0}\right) \equiv \sup _{(T, W, V, C)} E\left[\int_{0}^{\infty} e^{-\rho t} u\left(C_{t}\right) d t\right]
$$

subject to

$$
\begin{gathered}
\int_{\tau_{k}}^{\tau_{k+1}} C_{t} d t=(1-a) W\left(\tau_{k}\right)-b, \quad k=1,2,3, \ldots \\
X\left(\tau_{k+1}\right)=(1-\epsilon)\left(X_{\tau_{k}}-W_{\tau_{k}}\right) e^{r T_{k}}+V_{\tau_{k}}\left(\Gamma_{k+1}-e^{r T_{k}}\right) \geq 0, \quad k=1,2,3, \ldots
\end{gathered}
$$

Since

$$
E\left[\int_{0}^{\infty} e^{-\rho t} u\left(C_{t}\right) d t\right]=E\left[\sum_{k=1}^{\infty} e^{-\rho \tau_{k}} \int_{\tau_{k}}^{\tau_{k+1}} e^{-\rho\left(t-\tau_{k}\right)} u\left(C_{t}\right) d t\right]
$$

we can solve (8)-(10) in two steps. In the first step, we solve the control problem for consumption between transaction intervals given any feasible $(T, W, V)$. This is a deterministic continuous-time control problem because $C$ is adapted to the filtration H.

For an investor with horizon $t$ and initial money endowment $y$, the deterministic control problem for consumption is

$$
K(t, y) \equiv \sup _{\left\{C_{s}: 0 \leq s \leq t\right\}} \int_{0}^{t} e^{-\rho s} u\left(C_{s}\right) d s
$$

subject to

$$
\int_{0}^{t} C_{s} d s \leq y
$$


In the second step, the investor chooses a budget feasible $(T, W, V)$ to maximize $E\left[\sum_{k=1}^{\infty} e^{-\rho \tau_{k}} K\left(T_{k}, M_{\tau_{k}}\right)\right]$. This step is similar to a stochastic discrete-time control problem except that the sequence $T$ of transaction intervals is controllable.

We solve the deterministic control problem (11)-(12) in Appendix 1 and report the corresponding value function $K$ in Lemma 2 .

Lemma 2. The optimal value function $K(t, y)$ for the deterministic control problem (11)-(12) is

$$
K(t, y)=\left(\frac{1-\alpha}{\rho}\right)^{1-\alpha}\left[1-\exp \left(\frac{-\rho}{1-\alpha} t\right)\right]^{1-\alpha} \frac{1}{\alpha} y^{\alpha}
$$

Hence, for $k=1,2,3, \ldots$,

$$
K\left(T_{k}, M_{\tau_{k}}\right)=(1-a)^{\alpha}\left(\frac{1-\alpha}{\rho}\right)^{1-\alpha}\left[1-\exp \left(\frac{-\rho}{1-\alpha} T_{k}\right)\right]^{1-\alpha} \frac{1}{\alpha}\left(W_{\tau_{k}}-b_{1}\right)^{\alpha}
$$

where $b_{1}=\frac{b}{1-a}>0$.

PROOF: Please refer to Appendix 1.

Since $\alpha$ and $a$ are less than one, we can leave out the term $(1-a)^{\alpha}(1-\alpha)^{1-\alpha} \rho^{\alpha-1}$ in the following discussion. When $b=0$, the coefficient $a$ of proportional withdrawal costs does not affect the consumption-portfolio decision. This is a special feature of investors with constant relative risk aversion.

In general, the optimal transaction intervals are random, with the length of each interval depending non-trivially on the total wealth at the beginning of each interval. We will first proceed with a conjecture that the value function is wellbehaved and that there is some transactions interval $N$ such that it is optimal to choose $T_{1}=T_{2}=\cdots=N>0$. Later, we can confirm this conjecture in the case $b=0$. It is fairly plain that the conjecture is false for $b \neq 0$.

By (5), the returns $\Gamma_{2}, \Gamma_{3}, \ldots$ are i.i.d. and equivalent in distribution to

$$
\Lambda_{N} \equiv \Gamma_{2}=\exp \left[\left(\mu-\frac{1}{2} \sigma^{2}\right) N+\sigma B_{N}\right]
$$


Let $Q_{N} \equiv 1-e^{\frac{-\rho N}{1-\alpha}}$. From (13), the modified control problem is

$$
\mathbf{U}\left(X_{0}\right)=\sup _{\{N>0, W \in \mathbf{W}, V \in \mathbf{V}\}} E\left[\sum_{k=1}^{\infty} e^{-\rho \tau_{k}} Q_{N}^{1-\alpha} \frac{1}{\alpha}\left(W_{\tau_{k}}-b_{1}\right)^{\alpha}\right]
$$

subject to

$$
X_{\tau_{k+1}}=(1-\epsilon)\left(X_{\tau_{k}}-W_{\tau_{k}}\right) e^{r N}+V_{\tau_{k}}\left(\Gamma_{k+1}-e^{r N}\right) \geq 0
$$

Of course $\tau_{k}=(k-1) N$. The Bellman principle implies that, for $k=1,2,3, \ldots$,

$$
\mathbf{U}\left(X_{\tau_{k}}\right)=\sup _{\left(W_{\tau_{k}}, V_{\tau_{k}}, N\right)}\left\{Q_{N}^{1-\alpha} \frac{1}{\alpha}\left(W_{\tau_{k}}-b_{1}\right)^{\alpha}+e^{-\rho N} E\left[\mathbf{U}\left(X_{\tau_{k+1}}\right) \mid \mathcal{H}_{\tau_{k}}\right]\right\} .
$$

Assuming differentiability and technical conditions, the necessary conditions for (16) are

$$
\begin{aligned}
Q_{N}^{1-\alpha}\left(W_{\tau_{k}}-b_{1}\right)^{\alpha-1} & =(1-\epsilon) e^{-(\rho-r) N} E\left[\mathbf{U}^{\prime}\left(X_{\tau_{k+1}}\right) \mid \mathcal{H}_{\tau_{k}}\right], \\
E\left[\mathbf{U}^{\prime}\left(X_{\tau_{k+1}}\right)\left(\Lambda_{N}-e^{r N}\right) \mid \mathcal{H}_{\tau_{k}}\right] & =0 \\
\frac{1}{\alpha} \rho Q_{N}^{-\alpha} e^{\frac{-\rho N}{1-\alpha}}\left(W_{\tau_{k}}-b_{1}\right)^{\alpha} & =e^{-\rho N}\left(\rho E\left[\mathbf{U}\left(X_{\tau_{k+1}}\right) \mid \mathcal{H}_{\tau_{k}}\right]-\frac{\partial E\left[\mathbf{U}\left(X_{\tau_{k+1}}\right) \mid \mathcal{H}_{\tau_{k}}\right]}{\partial N}\right) .
\end{aligned}
$$

A proof similar to that of Theorem 1 by Hakansson (1970) shows that, given $N$, the optimal value function and unique solution to (16) satisfy, for $k=1,2,3, \ldots$,

$$
\begin{aligned}
\mathbf{U}\left(X_{\tau_{k}}\right) & =Q_{N}^{1-\alpha} A_{N}^{\alpha-1} \frac{1}{\alpha}\left(X_{\tau_{k}}-Y_{N}\right)^{\alpha}, \\
W_{\tau_{k}} & =A_{N}\left(X_{\tau_{k}}-Y_{N}\right)+b_{1}, \\
V_{\tau_{k}} & =(1-\epsilon)\left(X_{\tau_{k}}-Y_{N}\right)\left(1-A_{N}\right) \Pi_{N},
\end{aligned}
$$

where

$$
\begin{aligned}
A_{N} & =1-\left[(1-\epsilon)^{\alpha} e^{-\rho N} \Omega_{N}\right]^{\frac{1}{1-\alpha}}, \\
Y_{N} & =\frac{b_{1}(1-\epsilon) e^{r N}}{(1-\epsilon) e^{r N}-1}
\end{aligned}
$$


and where $\Omega_{N}$ and $\Pi_{N}$ are defined by the optimization problem:

$$
\Omega_{N} \equiv E\left(\left[e^{r N}+\Pi_{N}\left(\Lambda_{N}-e^{r N}\right)\right]^{\alpha}\right) \equiv \sup _{\{\pi\}} E\left(\left[e^{r N}+\pi\left(\Lambda_{N}-e^{r N}\right)\right]^{\alpha}\right)
$$

subject to

$$
\mathcal{P}\left(e^{r N}+\pi\left(\Lambda_{N}-e^{r N}\right) \geq 0\right)=1
$$

(Appendix 2 shows that $\Omega_{N}$ and $\Pi_{N}$ are well-defined.)

We will derive an equation for $N$ and later confirm that a solution is the optimal choice for $N$. Based on equation (6), we show in Appendix 3 that (at least for the relevant case $b=0$ )

$$
\begin{aligned}
& \frac{\partial E\left[\mathbf{U}\left(X_{\tau_{k+1}}\right) \mid \mathcal{H}_{\tau_{k}}\right]}{\partial N} \\
= & E\left[\left[r\left(X_{\tau_{k+1}}-V_{\tau_{k+1}}\right)+\mu V_{\tau_{k+1}}\right] \mathbf{U}^{\prime}\left(X_{\tau_{k+1}}\right)+\frac{1}{2} \sigma^{2} V_{\tau_{k}}^{2} \mathbf{U}^{\prime \prime}\left(X_{\tau_{k+1}}\right) \mid \mathcal{H}_{\tau_{k}}\right] .
\end{aligned}
$$

After substituting (18)-(23) into (17), tedious simplification yields

$$
\left(X_{\tau_{k}}-Y_{N}\right) h(N)=e^{-\rho N} r Y_{N} \alpha Q_{N}\left[(1-\epsilon)\left(1-A_{N}\right)\right]^{\alpha-1} E\left(\left[e^{r N}+\Pi_{N}\left(\Lambda_{N}-e^{r N}\right)\right]^{\alpha-1}\right)
$$

where

$$
\begin{gathered}
h(N)=\rho e^{\frac{-\rho N}{1-\alpha}} A_{N}+\left(1-A_{N}\right) Q_{N}\left[\alpha r+\alpha(1-\epsilon)(\mu-r)\left(1-A_{N}\right) \Pi_{N}\right. \\
\left.-\frac{1}{2} \sigma^{2} \alpha(1-\epsilon)^{2}(1-\alpha)\left(1-A_{N}\right)^{2} \Pi_{N}^{2}-\rho\right] .
\end{gathered}
$$

Equation (24) has only one unknown variable, $N$. With $b \neq 0$ (and accordingly, $\left.Y_{N} \neq 0\right)$, the right hand side of $(24)$ is not equal to zero. Then either equation (24) has no solution or the solution depends non-trivially on $X_{\tau_{k}}$. In either case, there is a contradiction to fixed transactions intervals with $b \neq 0$. With $b=0$, however, we will show the existence of a solution in $N$ to (24).

So far, we have implicitly assumed that the optimal value function $\mathbf{U}\left(X_{\tau_{k}}\right)$ in (18) is finite, meaning that the control problem (14)-(16) has a solution given $N>0$. 
This is equivalent to $A_{N}>0$, or $(1-\epsilon)^{\alpha} e^{-\rho N} \Omega_{N}<1$. A sufficient condition ${ }^{1}$ is

$$
\rho>\alpha r+\alpha 1_{\{\mu \geq r\}}(\mu-r)=\max (\alpha \mu, \alpha r),
$$

where $1_{\{\cdot\}}$ is the indicator function. For $\mu \geq r$, which is natural, this condition is merely $\rho>\alpha \mu$.

Lemma 3. Suppose $b=0$ and $\rho>\max (\alpha \mu, \alpha r)$. Then the equation in $N$ defined by (24) has a solution.

PROOF: Since

$$
\exp [N(\alpha r-\rho)] \leq e^{-\rho N} \Omega_{N} \leq \exp (N[\max (\alpha \mu, \alpha r)-\rho])
$$

we have $\lim _{N \rightarrow 0} e^{-\rho N} \Omega_{N}=1$ and $\lim _{N \rightarrow \infty} e^{-\rho N} \Omega_{N}=0$. Recalling that $Q_{N}=$ $1-e^{\frac{-\rho N}{1-\alpha}}$

$$
\lim _{N \rightarrow 0} h(N)=\rho\left[1-(1-\epsilon)^{\frac{\alpha}{1-\alpha}}\right]>0 .
$$

On the other hand,

$$
\begin{aligned}
h(N) & \leq \rho e^{\frac{-\rho N}{1-\alpha}} A_{N}+\left(1-A_{N}\right) Q_{N}\left[\alpha r+\alpha(1-\epsilon)(\mu-r)\left(1-A_{N}\right) \Pi_{N}-\rho\right] \\
& \leq \rho e^{\frac{-\rho N}{1-\alpha}} A_{N}+\left(1-A_{N}\right) Q_{N}[\max (\alpha \mu, \alpha r)-\rho] .
\end{aligned}
$$

The last inequality follows from the fact that $A_{N} \in(0,1)$ and $\Pi_{N} \in[0,1]$. Letting $N \rightarrow \infty$, we have

$$
\lim _{N \rightarrow \infty} h(N) \leq \max (\alpha \mu, \alpha r)-\rho<0 .
$$

1 A necessary and sufficient condition for (22) is $\pi \in[0,1]$. From (21), Jensen's inequality yields, for $\alpha \in(0,1)$,

$$
\begin{aligned}
\alpha e^{-\rho N} \Omega_{N} & \leq e^{-\rho N}\left[e^{r N}+\Pi_{N}\left(E\left(\Lambda_{N}\right)-e^{r N}\right)\right]^{\alpha} \\
& =e^{-\rho N}\left[e^{r N}+\Pi_{N}\left(e^{\mu N}-e^{r N}\right)\right]^{\alpha} \\
& =e^{-\rho N}\left[e^{r N}+1_{\{\mu \geq r\}}\left(e^{\mu N}-e^{r N}\right)\right]^{\alpha} \\
& =\exp \left[\alpha r+\alpha 1_{\{\mu \geq r\}}(\mu-r)-\rho\right] \\
& <1, \quad \text { if } \rho>\alpha r+\alpha 1_{\{\mu \geq r\}}(\mu-r) .
\end{aligned}
$$


If $h(N)$ is continuous in $N$, there exists therefore some $\bar{N}$ such that $h(\bar{N})=0$. It remains to show continuity of $h$. The only point to verify is continuity of $\Pi_{N}$ as a function of $N$, which follows from the first order conditions of (21) and the implicit function theorem.

Lemma 4. Let $\bar{\alpha}(k) \equiv\left(1-e^{\frac{-\rho T_{k}}{1-\alpha}}\right)^{1-\alpha}$. Suppose that $f$ is a real-valued function on $[0, \infty)$ satisfying the two conditions:

(i) For all $k$,

$$
f\left(X_{\tau_{k}}\right)=\sup _{\left(W_{\tau_{k}}, V_{\tau_{k}}, T_{k}\right)}\left\{\bar{\alpha}(k) \frac{1}{\alpha}\left(W_{\tau_{k}}-b_{1}\right)^{\alpha}+E\left[e^{-\rho T_{k}} f\left(X_{\tau_{k+1}}\right) \mid X_{\tau_{k}}\right]\right\} .
$$

(ii) For any feasible policy,

$$
\lim _{k \rightarrow \infty} E\left[e^{-\rho \tau_{k}} f\left(X_{\tau_{k}}\right)\right]=0
$$

If $\left(W_{\tau_{k}}^{*}, V_{\tau_{k}}^{*}, T_{k}^{*}\right)$ achieves the maximum in (27) for all $k$, then $f$ is the value function for the control problem (8)-(10), and $\left\{W_{\tau_{k}}^{*}, V_{\tau_{k}}^{*}, T_{\tau_{k}}^{*}\right\}$ is an optimal policy.

PROOF: We have:

$$
\begin{aligned}
f\left(X_{0}\right) & =\sup _{\left(W_{0}, V_{0}, T_{1}\right)}\left\{\bar{\alpha}(1) \frac{1}{\alpha}\left(W_{0}-b_{1}\right)^{\alpha}+e^{-\rho T_{1}} E\left[f\left(X_{\tau_{2}}\right)\right]\right\} \\
& \geq \bar{\alpha}(1) \frac{1}{\alpha}\left(W_{0}-b_{1}\right)^{\alpha}+e^{-\rho T_{1}} E\left[f\left(X_{\tau_{2}}\right)\right]
\end{aligned}
$$

for any feasible $W_{0}, V_{0}$, and $T_{1}$. By induction, for any $(W, V, T) \in \mathbf{W} \times \mathbf{V} \times \mathbf{T}$ and any $k$,

$$
f\left(X_{0}\right) \geq E\left[\sum_{i=1}^{k} e^{-\rho \tau_{i}} \bar{\alpha}(i) \frac{1}{\alpha}\left(W_{\tau_{i}}-b_{1}\right)^{\alpha}+e^{-\rho \tau_{k+1}} f\left(X_{\tau_{k+1}}\right)\right] .
$$

Let $k \rightarrow \infty$, it follows by condition (ii) that

$$
f\left(X_{0}\right) \geq E\left[\sum_{k=1}^{\infty} e^{-\rho \tau_{k}} \bar{\alpha}(k) \frac{1}{\alpha}\left(W_{\tau_{k}}-b_{1}\right)^{\alpha}\right]
$$

This holds for an arbitrary feasible policy $(T, W, V)$, so $f\left(X_{0}\right) \geq \mathbf{U}\left(X_{0}\right)$. On the other hand, $\mathbf{U}\left(X_{0}\right) \geq f\left(X_{0}\right)$ by the definition of $\mathbf{U}\left(X_{0}\right)$. We have $f\left(X_{0}\right)=\mathbf{U}\left(X_{0}\right)$ and, consequently, $\left\{W^{*}, V^{*}, T^{*}\right\}$ is an optimal policy. 
Theorem. Suppose $b=0$ and $\rho>\max (\alpha \mu, \alpha r)$. An optimal policy $(T, V, W, C)$ exists and the optimal transaction intervals $\left\{T_{k}\right\}$ are equal constants.

PROOF: With $b=0$, equation (24) is equivalent to $h(N)=0$. By Lemma 3, there exists a scalar $\bar{N}>0$ such that $h(\bar{N})=0$. Consider the following withdrawal and investment policy, and function $f$ :

$$
\begin{aligned}
f\left(X_{\tau_{k}}\right) & =Q_{\bar{N}}^{1-\alpha} A_{\bar{N}}^{\alpha-1} \frac{1}{\alpha} X_{\tau_{k}}^{\alpha} \\
W_{\tau_{k}} & =A_{\bar{N}} X_{\tau_{k}} \\
V_{\tau_{k}} & =(1-\epsilon)\left(1-A_{\bar{N}}\right) \Pi_{\bar{N}} X_{\tau_{k}} \\
T_{k} & =\bar{N} .
\end{aligned}
$$

From the proof of Theorem 1, we know that $f\left(X_{\tau_{k}}\right)$ satisfies $(27)$ and $\left(W_{\tau_{k}}, V_{\tau_{k}}, T_{\tau_{k}}\right)$ achieves the maximum in (27) for all $k$. Letting $K_{\bar{N}} \equiv \frac{1}{\alpha} Q_{\bar{N}}^{1-\alpha} A_{\bar{N}}^{\alpha-1}$,

$$
\begin{aligned}
E\left[e^{-\rho \tau_{k+1}} f\left(X_{\tau_{k+1}}\right)\right] & =e^{-\rho k \bar{N}} K_{\bar{N}} E\left(X_{\tau_{k+1}}^{\alpha}\right) \\
& \leq e^{-\rho k \bar{N}} K_{\bar{N}} E_{\{W \equiv 0\}}\left(X_{\tau_{k+1}}^{\alpha}\right) \\
& \leq e^{-\rho k \bar{N}} K_{\bar{N}}(1-\epsilon)^{\alpha} \Omega_{\bar{N}} E_{\{W \equiv 0\}}\left(X_{\tau_{k}}^{\alpha}\right) \\
& \leq e^{-\rho k \bar{N}} K_{\bar{N}} X_{0}^{\alpha} \prod_{i=1}^{k+1}(1-\epsilon)^{\alpha} \Omega_{\bar{N}} \\
& =K_{\bar{N}} X_{0}^{\alpha}\left[(1-\epsilon)^{\alpha} \Omega_{\bar{N}} e^{-\rho \bar{N}}\right]^{k+1},
\end{aligned}
$$

where $E_{\{W \equiv 0\}}$ denotes expectation under the zero-money-withdrawal policy. The second and the third inequalities above follow by (21) and (29). Since $(1-\epsilon)^{\alpha} \Omega_{\bar{N}} e^{-\rho \bar{N}}<$ 1 , for any feasible policy, (28) is satisfied. By Lemma 4, the theorem has been proved.

With proportional transactions costs and portfolio management fees, the continuoustime consumption and portfolio problem for an investor with constant relative risk aversion is therefore equivalent to a deterministic continuous-time control problem for consumption between transaction intervals combined with a stochastic discretetime portfolio selection problem. 


\section{EXTENSION}

Let $\bar{B}=\left(B^{(1)}, \ldots, B^{(L)}\right)$ be an $L$-dimensional ${ }^{2}$ Standard Brownian Motion, and let $\bar{S}=\left(S^{(1)}, \ldots, S^{(m)}\right)$ and $\bar{D}=\left(D^{(1)}, \ldots, D^{(m)}\right)$ denote the vector of price processes and the vector of cumulative dividend processes of the $m$ risky securities. We assume that $\bar{S}$ and $\bar{D}$ satisfy

$$
G_{t}^{(i)} \equiv S_{t}^{(i)}+D_{t}^{(i)}=1+\int_{0}^{t} S_{s}^{(i)} \mu_{i} d s+\int_{0}^{t} S_{s}^{(i)} \bar{\sigma}_{i}^{\prime} d \bar{B}_{s}, \quad t \geq 0, \quad i=1,2, \ldots m
$$

for given scalars $\mu_{i}$ and vectors $\bar{\sigma}_{i} \in \mathbb{R}^{L}$. Let $\bar{\mu}=\left(\mu_{1}, \ldots, \mu_{m}\right)$ and $\bar{\sigma}=\left(\bar{\sigma}_{1}, \ldots, \bar{\sigma}_{m}\right)$. The price process of the riskless security is given by (2) in Section II.

We use the notation defined in Section II, modifying scalars to vectors where necessary. The market value at time $\tau_{k+1}$ of a unit of wealth invested in the $i$-th security at time $\tau_{k}$, with interim dividends continually re-invested, is

$$
\Gamma_{k+1}^{(i)}=\exp \left[\left(\mu_{i}-\frac{1}{2} \bar{\sigma}_{i}^{\prime} \bar{\sigma}_{i}\right) T_{k}+\bar{\sigma}_{i}^{\prime}\left(\bar{B}_{\tau_{k+1}}-\bar{B}_{\tau_{k}}\right)\right], \quad i=1,2, \ldots m
$$

The total wealth in securities at the $(k+1)$-th transaction is

$$
X_{\left(\tau_{k+1}\right)}=(1-\epsilon)\left(X_{\tau_{k}}-W_{\tau_{k}}\right)+\int_{\tau_{k}}^{\tau_{k+1}}\left[r\left(X_{t}+\bar{V}_{t}^{\prime}(\bar{\mu}-r \overline{1})\right] d t+\int_{\tau_{k}}^{\tau_{k+1}} \bar{V}_{t}^{\prime} \bar{\sigma} d \bar{B}_{t}\right.
$$

where $\overline{1}$ is a $m$-dimensional vector with all elements equal to 1 .

The control problem corresponding to Theorem 1 is:

$$
\mathbf{U}\left(X_{0}\right) \equiv \sup _{\{N>0, W, \bar{V}\}} E\left[\sum_{k=1}^{\infty} e^{-\rho \tau_{k}} Q_{N}^{1-\alpha} \frac{1}{\alpha}\left(W_{\tau_{k}}-b_{1}\right)^{\alpha}\right]
$$

subject to

$$
X_{\left(\tau_{k+1}\right)}=(1-\epsilon)\left(X_{\tau_{k}}-W_{\tau_{k}}\right) e^{r N}+\bar{V}_{\tau_{k}}^{\prime}\left(\bar{\Gamma}_{k+1}-e^{r N} \overline{1}\right) \geq 0
$$

2 In this section, a vector or a matrix will be denoted by a bar above the character, and $\bar{B}^{\prime}$ is the transpose of $\bar{B}$. All vectors are column vectors. 
Given any $N>0$, arguments similar to those made in the proof of Theorem 1 show that, for each $k$, an optimal policy must satisfy

$$
\begin{aligned}
\mathbf{U}\left(X_{\tau_{k}}\right) & =Q_{N}^{1-\alpha}\left(A_{N}\right)^{\alpha-1} \frac{1}{\alpha}\left(X_{\tau_{k}}-Y_{N}\right)^{\alpha}, \\
W_{\tau_{k}} & =A_{N}\left(X_{\tau_{k}}-Y_{N}\right)+b_{1}, \\
\bar{V}_{\tau_{k}} & =(1-\epsilon)\left(X_{\tau_{k}}-Y_{N}\right)\left(1-A_{N}\right) \bar{\Pi}_{N}, \\
\left(X_{\tau_{k}}-Y_{N}\right) h(N) & =e^{-\rho N} r Y_{N} \alpha Q_{N}\left[(1-\epsilon)\left(1-A_{N}\right)\right]^{\alpha-1} E\left(\left[e^{r N}+\bar{\Pi}_{N}^{\prime}\left(\bar{\Lambda}_{N}-e^{r N} \overline{1}\right)\right]^{\alpha-1}\right),
\end{aligned}
$$

where

$$
\begin{aligned}
h(N)=\rho e^{\frac{-\rho N}{1-\alpha}} A_{N}+\left(1-A_{N}\right) Q_{N}\left[\alpha r+\alpha(1-\epsilon)\left(1-A_{N}\right)(\bar{\mu}-r \overline{1})^{\prime} \bar{\Pi}_{N}\right. & \\
& \left.\quad-\frac{1}{2} \alpha(1-\alpha)(1-\epsilon)\left(1-A_{N}\right)^{2} \bar{\Pi}_{N}^{\prime} \overline{\sigma \sigma}^{\prime} \bar{\Pi}_{N}-\rho\right], \\
A_{N}= & 1-\left[(1-\epsilon)^{\alpha} e^{-\rho N} \Omega_{N}\right]^{\frac{1}{1-\alpha}} \\
Y_{N}= & \frac{b_{1}(1-\epsilon) e^{r N}}{(1-\epsilon) e^{r N}-1}
\end{aligned}
$$

and where $\Omega_{N}$ and $\Pi_{N}$ are defined by the optimization problem:

$$
\Omega_{N} \equiv E\left(\left[e^{r N}+\bar{\Pi}_{N}^{\prime}\left(\bar{\Lambda}_{N}-e^{r N} \overline{1}\right)\right]^{\alpha}\right) \equiv \sup _{\{\bar{\pi}\}} E\left(\left[e^{r N}+\bar{\pi}^{\prime}\left(\bar{\Lambda}_{N}-e^{r N} \overline{1}\right)\right]^{\alpha}\right),
$$

subject to

$$
\mathcal{P}\left(e^{r N}+\bar{\pi}^{\prime}\left(\bar{\Lambda}_{N}-e^{r N \overline{1}}\right) \geq 0\right)=1 .
$$

Similarly, it follows from Appendix 2 that $\Omega_{N}$ and $\Pi_{N}$ are well-defined.

By analogy with (26), we assume that

$$
\rho>\alpha r+\alpha \sum_{i=1}^{m} 1_{\left\{\mu_{i} \geq r\right\}}\left(\mu_{i}-r\right)
$$

in order to guarantee a solution for the control problem. All the arguments made in Lemma 3, Lemma 4, and the theorem are valid with condition (26) replaced by (31). Hence, with withdrawal costs proportional to total wealth, the optimal transaction intervals are equal constants for an investor with constant risk aversion. 


\section{APPENDIX 1}

We prove that the investor will not withdraw more money than the amount needed for financing consumption.

Lemma 1. An optimal policy $(T, W, V, C)$ must satisfy

$$
\int_{\tau_{k}}^{\tau_{k+1}} C_{t} d t=(1-a) W_{\tau_{k}}-b, \quad k=1,2,3 \ldots
$$

PROOF: Suppose there exists an interval $\left[\tau_{j}, \tau_{j+1}\right)$ such that

$$
\int_{\tau_{j}}^{\tau_{j+1}} C_{t} d t<(1-a) W_{\tau_{j}}-b
$$

Let $d=(1-a) W_{\tau_{j}}-b-\int_{\tau_{j}}^{\tau_{j+1}} C_{t} d t>0$. The investor will be better off if he or she invests the extra amount of money $d$ in the riskless security from $\tau_{j}$ to $\tau_{j+1}$, and consumed the increased interest income $d\left(e^{r T_{j}}-1\right)$ in the next interval. That is, the policy $(T, W, V, C)$ is strictly dominated by a feasible policy $(T, \bar{W}, V, \bar{C})$ defined by:

$$
\begin{aligned}
\bar{W}_{\tau_{j}} & =\frac{1}{1-a}\left[\int_{\tau_{j}}^{\tau_{j+1}} C_{t} d t+b\right], \\
\bar{W}_{\tau_{j+1}} & =W_{\tau_{j+1}}+d e^{r T_{j}}>W_{\tau_{j+1}}, \\
\bar{C}_{t} & =C_{t}+\frac{1}{T_{j+1}} d\left(e^{r T_{j}}-1\right)>C_{t}, \quad t \in\left[\tau_{j+1}, \tau_{j+2}\right), \\
\bar{C}_{t} & =C_{t}, \bar{W}_{\tau_{k}}=W_{\tau_{k}}, \quad \text { otherwise. }
\end{aligned}
$$

This contradicts the fact that $(T, W, V, C)$ is optimal. Hence, for all $k$,

$$
\int_{\tau_{k}}^{\tau_{k+1}} C_{t} d t \geq(1-a) W_{\tau_{k}}-b
$$

On the other hand, since consumption expenditure must be financed from the stock of money,

$$
\sum_{i=1}^{k} \int_{\tau_{i}}^{\tau_{i+1}} C_{t} d t \leq \sum_{i=1}^{k}\left[(1-a) W_{\tau_{i}}-b\right], \quad k=1,2,3, \ldots
$$

An optimal policy must therefore satisfy $\int_{\tau_{k}}^{\tau_{k+1}} C_{t} d t=(1-a) W_{\tau_{k}}-b$ for all $k$. 
Next, we solve the deterministic continuous control problem (11)-(12) for consumption. Let

$$
\begin{gathered}
y_{s} \equiv y-\int_{0}^{t-s} C_{\tau} d \tau . \\
K\left(s, y_{s}\right) \equiv \sup _{\left\{C_{\tau}: s \leq \tau \leq t\right\}} \int_{0}^{s} e^{-\rho(\tau-s)} \frac{1}{\alpha} C_{\tau}^{\alpha} d \tau .
\end{gathered}
$$

The Bellman equation for (11)-(12) is

$$
0=\sup _{\left(C_{s}\right)}\left\{\frac{1}{\alpha} C_{s}^{\alpha}+K_{1}\left(s, y_{s}\right)+K_{2}\left(s, y_{s}\right)\left(-C_{s}\right)\right\},
$$

where $K_{i}\left(s, y_{s}\right), i=1,2$, denotes the partial derivative of $K\left(s, y_{s}\right)$ with respect to the $i$-th argument. The first order necessary condition of (A1) is

$$
C_{s}^{\alpha-1}=K_{2}\left(s, y_{s}\right)
$$

Substituting (A2) into (A1), we have the partial differential equation

$$
(1-\alpha) K_{2}^{\frac{\alpha}{\alpha-1}}\left(s, y_{s}\right)+\alpha K_{1}\left(s, y_{s}\right)=0
$$

with boundary condition $K\left(0, y_{t}\right)=0$. The solution to (A3) is

$$
K\left(s, y_{s}\right)=\left(\frac{1-\alpha}{\rho}\right)^{1-\alpha}\left[1-\exp \left(\frac{-\rho s}{1-\alpha}\right)\right]^{1-\alpha} \frac{1}{\alpha} y_{s}^{\alpha} .
$$

From (A2) and (A4),

$$
C_{s}^{*}=\left(\frac{1-\alpha}{\rho}\left[1-\exp \left(\frac{-\rho s}{1-\alpha}\right)\right]\right)^{-1} y_{s} .
$$

The Verification Theorem (for example, Theorem 4.4 of Fleming and Rishel (1975)) says that if a real-valued function $f$ on $\mathbb{R}_{+} \times \mathbb{R}_{+}$satisfies (A1) and the process $C^{*}$ achieves the maximum of (A1) with $K\left(s, y_{s}\right)$ being replaced by $f\left(s, y_{s}\right)$, then $f(t, y)=K(t, y)$ and $C^{*}$ is an optimal consumption strategy to (13)-(14). Clearly, $K\left(s, y_{s}\right)$ defined by (A4) satisfies (A1) and is strictly concave. Consequently, $C^{*}$ defined by (A5) is the unique maximizing process to (A1). We have (13) by the Verification Theorem.

\section{APPENDIX 2}


Suppose $r$ is a scalar strictly greater than 1 , and $\beta_{1}, \beta_{2}, \ldots, \beta_{m}$ are positive real-valued random variables satisfying

$$
\mathcal{P}\left(\sum_{i=1}^{m}\left(\beta_{i}-r\right) \theta_{i}<0\right)>0 \text { for all finite } \theta_{1}, \theta_{2}, \ldots, \theta_{m}
$$

A lemma by Hakansson [(1970), p. 593] states that the following optimization problem has a unique solution, with value function

$$
J\left(v_{1}, \ldots, v_{m}\right) \equiv \sup _{\left(v_{1}, \ldots, v_{m}\right)} E\left(\left[\sum_{i=1}^{m}\left(\beta_{i}-r\right) v_{i}+r\right]^{\alpha}\right)
$$

subject to

$$
\mathcal{P}\left(\sum_{i=1}^{m}\left(\beta_{i}-r\right) v_{i}+r \geq 0\right)=1
$$

\section{APPENDIX 3}

From (6), Ito's lemma implies that

$$
\mathbf{U}\left[X_{\left(\tau_{k+1}\right)+}\right]=\mathbf{U}\left(X_{\tau_{k}}\right)+\int_{\tau_{k}}^{\tau_{k+1}} g(t) d t+\int_{\tau_{k}}^{\tau_{k+1}} \sigma \mathbf{U}^{\prime}\left(X_{t}\right) V_{t} d B_{t},
$$

where

$$
g(t) \equiv\left[r\left(X_{t}-V_{t}\right)+\mu V_{t}\right] \mathbf{U}^{\prime}\left(X_{t}\right)+\frac{1}{2} \sigma^{2} V_{t}^{2} \mathbf{U}^{\prime \prime}\left(X_{t}\right)
$$

If $\left\{Z_{t} \equiv \int_{\tau_{k}}^{t} \sigma \mathbf{U}^{\prime}\left(X_{s}\right) V_{s} d B_{s}: t \in\left(\tau_{k}, \tau_{k+1}\right]\right\}$ is a martingale, we have $E\left(Z_{\tau_{k+1}}\right)=$ $E\left(Z_{\tau_{k}}\right)=0$, and equation (23) follows. In order to see that $\left\{Z_{t}\right\}$ is a martingale, note that, for $t \in\left(\tau_{k}, \tau_{k+1}\right]$,

$$
X_{t}^{2(\alpha-1)} V_{t}^{2} \leq X_{\tau_{k}}^{2 \alpha}(1-\epsilon)^{2 \alpha}\left(1-A_{N}\right)^{2 \alpha} \Pi_{N}^{2} \gamma_{k}(t)^{2} \leq X_{\tau_{k}}^{2 \alpha} \gamma_{k}^{2 \alpha}(t)
$$

Thus

$$
E\left[\int_{\tau_{k}}^{t} X_{s}^{2(\alpha-1)} V_{s}^{2} d s\right]<\infty, \quad t \in\left(\tau_{k}, \tau_{k+1}\right],
$$

which implies that $\left\{Z_{t}\right\}$ is a martingale. 


\section{References}

K.L. Chung and R.J. Williams (1983). An Introduction to Stochastic Integration. (Birkhäuser, Boston).

G. Constantinides (1986). "Capital Market Equilibrium with Transactions Costs," Journal of Political Economy. 94, pp. 842-862.

M. Davis and A. Norman (1988). "Portfolio Selection with Transactions Costs," Unpublished. Imperial College, London, forthcoming: Mathematics of Operations Reseach.

W. Fleming and R. Rishel (1975). Deterministic and Stochastic Optimal Control. (Springer-Verlag, Berlin).

N. Hakansson (1970). "Optimal Investment and Consumption Strategies under Risk for a Class of Utility Functions," Econometrica. 38, pp. 587-607.

H. Leland (1985). "Option Pricing and Replication with Transactions Costs," Journal of Finance. 40, pp. 1283-1301.

R. Merton (1971). "Optimum consumption and portfolio rules in a continuous time model," Journal of Economic Theory. 3, pp. 373-413.

-(1973). "Erratum," Journal of Economic Theory. 6, pp. 213-214. 